\title{
RNA-seq analysis of galaninergic neurons from ventrolateral preoptic nucleus identifies expression changes between sleep and wake
}

Xiaofeng Guo ${ }^{1 \dagger}$, Xiaoling Gao ${ }^{2 \dagger}$, Brendan T. Keenan', Jingxu Zhu', Dimitra Sarantopoulou, , Jie Lian', Raymond J. Galante', Gregory R. Grant ${ }^{3,5}$ and Allan I. Pack ${ }^{1 *}$ (D)

\begin{abstract}
Background: Previous studies show that galanin neurons in ventrolateral preoptic nucleus (VLPO-Gal) are essential for sleep regulation. Here, we explored the transcriptional regulation of the VLPO-Gal neurons in sleep by comparing their transcriptional responses between sleeping mice and those kept awake, sacrificed at the same diurnal time.

Results: RNA-sequencing (RNA-seq) analysis was performed on eGFP(+) galanin neurons isolated using laser captured microdissection (LCM) from VLPO. Expression of Gal was assessed in our LCM eGFP(+) neurons via real time qPCR and showed marked enrichment when compared to LCM eGFP(-) cells and to bulk VLPO samples. Gene set enrichment analysis utilizing data from a recent single-cell RNA-seq study of the preoptic area demonstrated that our VLPO-Gal samples were highly enriched with galanin-expressing inhibitory neurons, but not galanin-expressing excitatory neurons. A total of 263 genes were differentially expressed between sleep and wake in VLPO-Gal neurons. When comparing differentially expressed genes in VLPO-Gal neurons to differentially expressed genes in a wake-active neuronal region (the medial prefrontal cortex), evidence indicates that both systemic and cell-specific mechanisms contribute to the transcriptional regulation in VLPO-Gal neurons. In both wake-active and sleep-active neurons, ER stress pathways are activated by wake and cold-inducible RNA-binding proteins are activated by sleep. In contrast, expression of DNA repair genes is increased in VLPO-Gal during wakefulness, but increased in wake-active cells during sleep.

Conclusion: Our study identified transcriptomic responses of the galanin neurons in the ventrolateral preoptic nucleus during sleep and sleep deprivation. Data indicate that VLPO contains mainly sleep-active inhibitory galaninergic neurons. The VLPO galanin neurons show responses to sleep and wake similar to wake-active regions, indicating these responses, such as ER stress and cold-inducible RNA-binding proteins, are systemic affecting all neuronal populations. Region-specific differences in sleep/wake responses were also identified, in particular DNA repair. Our study expands knowledge about the transcriptional response of a distinct group of neurons essential for sleep.
\end{abstract}

Keywords: Ventrolateral preoptic nucleus, Galaninergic neurons, Spontaneous sleep, Sleep deprivation, Next-generation RNA-sequencing

\footnotetext{
* Correspondence: pack@pennmedicine.upenn.edu

Xiaofeng Guo and Xiaoling Gao are co-lead authors.

${ }^{1}$ Division of Sleep Medicine/Department of Medicine, University of Pennsylvania Perelman School of Medicine, Philadelphia 19104, USA

Full list of author information is available at the end of the article
}

(C) The Author(s). 2020 Open Access This article is licensed under a Creative Commons Attribution 4.0 International License, which permits use, sharing, adaptation, distribution and reproduction in any medium or format, as long as you give appropriate credit to the original author(s) and the source, provide a link to the Creative Commons licence, and indicate if changes were made. The images or other third party material in this article are included in the article's Creative Commons. licence, unless indicated otherwise in a credit line to the material. If material is not included in the article's Creative Commons licence and your intended use is not permitted by statutory regulation or exceeds the permitted use, you will need to obtain permission directly from the copyright holder. To view a copy of this licence, visit http://creativecommons.org/licenses/by/4.0/ The Creative Commons Public Domain Dedication waiver (http://creativecommons.org/publicdomain/zero/1.0/) applies to the data made available in this article, unless otherwise stated in a credit line to the data. 


\section{Background}

The preoptic area (POA) of the hypothalamus, particularly the ventrolateral preoptic area (VLPO), is essential for sleep regulation [46]. Damage to POA causes insomnia-like sleep disturbances in rats and cats, and the severity of sleep disturbances are correlated to the degree of damage [23, 37, 54]. Single neuron recordings in POA, including VLPO, demonstrated elevated discharge during non-rapid eye movement (NREM) and rapid eye movement (REM) sleep compared to waking, and the degree of increase reflected sleep depth, indicating their possible involvement in sleep homeostasis $[27,52,53]$. Studies using c-Fos immunoreactivity to indicate recent neuronal activities demonstrated increased numbers of c-Fos positive neurons in VLPO following consolidated sleep compared to wake, and the number of c-Fos positive neurons increased with recovery sleep after sleep deprivation, and showed a positive correlation with the amount of sleep before sacrifice $[17,18,50]$. These sleep-active neurons, particularly from VLPO, project to histaminergic tuberomammillary nucleus (TMN), serotonergic dorsal raphe (DR), and noradrenergic locus coreleus (LC), and the majority are GABAergic inhibitory neurons, suggesting that during sleep they inhibit multiple monoamine arousal systems $[49,51]$.

Studies show that approximately $80 \%$ of the neurons that project to TMN from VLPO express galanin [49], and this is conserved across multiple mammalian species [16]. In a recent study, optogenetic and chemogenetic tools were applied to specifically activate or inhibit VLPO galanin neurons (VLPO-Gal), demonstrating that VLPO-Gal neurons were sleep-active and sleep-promoting [28]. Chung et al. had conflicting results regarding the function of VLPO-Gal neurons [9]; however, this is likely related to the optogenetic stimulation being at too high a frequency, resulting in depolarization block [28]. In the present study, we aimed to further characterize the galaninergic neurons in VLPO by examining their behavioral state dependent transcriptional regulation between sleep and wake.

Galanin neuron localization using immunohistochemistry is challenging because galanin peptide is not mainly located in the cell body, and requires the use of colchicine to block axonal transport. However, this induces cellular stress [29, 60]. Hence, we utilized $\mathrm{Tg}$ (Gal-EGFP)HX109Gsat mice that express eGFP (enhanced green fluorescent protein) under control of the galanin promoter to aid identification of galaninexpressing neurons in VLPO. Laser capturing microdissection (LCM) was used to isolate individual eGFPexpressing cells. We used our previous design [34], comparing gene expression at 3,6, 9, and $12 \mathrm{~h}$ after lights-on (7 AM) between sleeping mice and those kept awake by gentle handling [14]. The same study design has been used in multiple published transcriptomics studies, including surveying bulk tissues of multiple brain regions [cortex and hypothalamus [34]] and peripheral tissues [lung and heart [2]], as well as LCM isolated cholinergic neurons in basal forebrain using microarrays [42]. The same design was also used in a recent bulk tissue study of medial prefrontal cortex using RNA-seq [20]. The similarity of the approach used here and in these prior studies allowed us to compare genes differentially expressed between sleep and wake in VLPO-Gal neurons to genes found differentially regulated in different regions and cell-types.

\section{Results}

\section{LCM enriched galanin expressing neurons from VLPO}

Galanin neurons are located in a number of nuclei and sub-structures of VLPO in POA, in addition to the VLPO core. These include the dorsal and medial VLPO extensions, as well as the median, medial, and the periventricular preoptic nuclei, as described previously [28]. To preserve the RNA quality, a $3 \mathrm{~mm}$ brain slice containing the VLPO region was post-fixed with $4 \%$ formaldehyde on ice for 5-min. The short post-fix was sufficient for the GFP fluorescent detection in the VLPO core and the medial VLPO extension, but not in the dorsal extension of VLPO and beyond (Supplementary figure 1 in Additional file 1). eGFP(+) cells in VLPO core were dissected using LCM with care taken to avoid picking eGFP $(+)$ cells in the medial VLPO extension, as shown in Supplementary figure 2 (Additional file 1). To validate enrichment of galanin expressing neurons in our LCM samples, we compared expression of Gal among the $\operatorname{eGFP}(+)$ neurons from the VLPO core, eGFP(-) cells from the adjacent piriform cortex on the same sections, and the bulk VLPO tissue (Bregma + 0.4 to -0.1 , lateral $\pm 0.5 \mathrm{~mm}$ ). Significant differences among the three sample types were observed for expression of both Gal (Kruskal-Wallis exact $p$-value $=0.0005$ ) and Aldh1l1 ( $p=0.0038)$. As shown in Fig. 1a, expression of Gal in eGFP(+) samples showed a median fold-increase of 116.9 (95\% confidence interval [CI]: 59.5, 497.0; $p=$ 0.0286 ) when compared to VLPO bulk tissues, whereas the expression of Gal in eGFP(-) samples showed a median fold-reduction of 13.4 (95\% CI: 2.0, 34,509.4; $p=$ 0.0286) when compared to the VLPO bulk tissue. On the other hand, expression of Aldh1l1, a specific marker of astrocytes, was significantly decreased in both the eGPF $(+)$ (median $[95 \%$ CI $]$ fold-decrease $=72.5 \quad[4.4$, $386.2] ; p=0.0286$ ) and the eGFP(-) (median [95\% CI] fold-decrease $=301.0[179.4,1252.9] ; p=0.0286)$ samples when compared to the VLPO bulk tissue (Fig. 1b). Thus, results indicate effective enrichment of galanin expressing neurons and removal of contaminating astrocytes in our VLPO-Gal samples using LCM. 

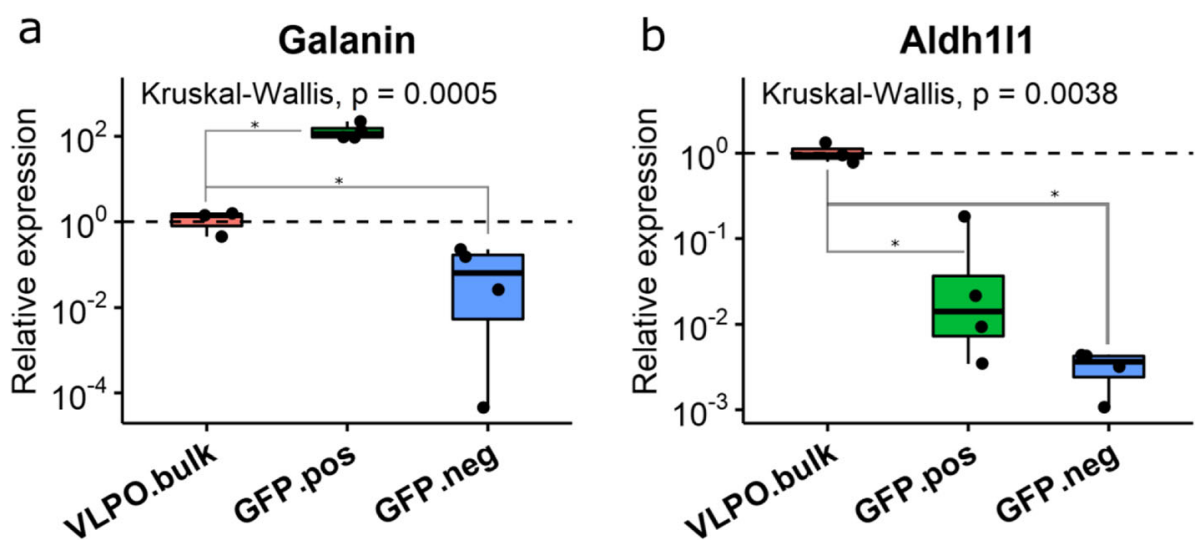

Fig. 1 Confirmation for the enrichment of galanin-expressing neurons in eGFP(+) samples collected using LCM. a eGFP(+) samples showed a median 116.9-fold increase in the expression of Gal when compared to the VLPO bulk tissues, indicating effective enrichment of galaninexpressing neurons in our eGFP(+) LCM samples. b Expression of the astrocyte gene Aldh1/1 in both the eGFP(+) and eGFP(-) samples was significantly lower when compared to the VLPO bulk tissues, indicating effective removal of contaminating astrocytes from the neuron samples collected by LCM. Kruskal-Wallis tests were made for comparisons among all three groups. Separate pairwise comparisons were then made between the VLPO bulk tissues and the LCM samples (eGFP+ and eGFP-) using one-tailed Wilcoxon exact test (see Methods). Y-axis shows fold difference in gene expression relative to VLPO bulk tissue on log10 scale

\section{Differentially regulated genes in VLPO-gal neurons between sleep and sleep deprivation}

We first examined the source of variation within the samples using unsupervised clustering of normalized gene expression data via multidimensional scaling (MDS) analysis. As shown in Fig. 2, samples from the spontaneous sleep mice (SS) were primarily separated from the samples from the sleep deprived mice (SDep) on the first dimension of the MDS plot, indicating behavioral state explains the greatest proportion of overall variability in the data. Samples collected at baseline (ZT0) fall between SS and SDep samples, which is consistent with the fact that this time point is prior to the onset of SS or SDep.

We next assessed differentially expressed genes (DEGs) in VLPO-Gal neurons between mice sleep deprived for 3, 6, 9 or $12 \mathrm{~h}$ starting from ZT0 and their time-matched SS controls using a moderated F-test in LimmaVoom. A total of 184 and 79 genes were up-regulated by SDep and SS, respectively, based on an FDR of 5\% (Fig. 3a). The SDep up-regulated genes were strongly enriched for GO biological process (BP) terms related to protein folding, including response to unfolded protein and response to ER stress, whereas the genes up-regulated during SS were enriched for nucleosome assembly, circadian rhythm, and positive regulation of translation (Fig. 3b). In the molecular function (MF) and cellular component (CC) categories, among the genes up-regulated by SDep, unfolded protein binding, chaperone binding, and poly(A) RNA binding were the most significantly enriched MF terms, and nucleus and ER chaperone complex were the most significantly enriched CC terms. Among the genes up-regulated by SS, DNA-binding and nucleosome were significantly enriched MF and CC terms. These results agree with the results obtained using the BP category. The complete list of differentially expressed genes and DAVID gene ontology analysis results are provided in Additional file 2.
Temporal changes in gene expression during sleep and sleep deprivation

We next incorporated the mice collected at ZT0 and evaluated the gene expression changes with time (from ZT0 to ZT12) during SS and SDep, separately. Among the 263 DEGs differentially regulated between SS and SDep, 7 genes were significantly associated with time during SS and 37 genes were significantly correlated with time during SDep at an FDR cutoff of 5\% (Table 1). The three circadian-regulated transcription factors, $D b p, Z b t b 16$, and Fkbp5, changed with time in the same direction during both SS and SDep, suggesting that SDep affected the magnitude of the circadian gene expression, but not the direction of the circadian phase. For the rest of the genes, the direction of the associations matched the behavior state-regulated gene expression changes. For example, Rbm3 was down-regulated by SDep when compared to the time-matched SS controls, and its expression associated positively with time during SS and negatively with time during SDep (Table 1).

When expanding the correlation tests to all detected transcripts, 3 genes (Nr1d2, Dbp , and Tef) showed significant association with time during SS and 17 genes (including Klf9, Tsc22d3, and Zbtb16; see Additional file 3 for full list) showed significant association with time during SDep at an FDR cutoff of 5\%. Although different individual genes were identified as being significantly correlated with time during SS and SDep, gene set enrichment analyses using the ranking of the correlation coefficients of all genes against the MSigDB database revealed positive enrichment of the pathways related to the circadian system and the circadian regulation of transcription for temporal changes during both SS and SDep (Additional file 3). 


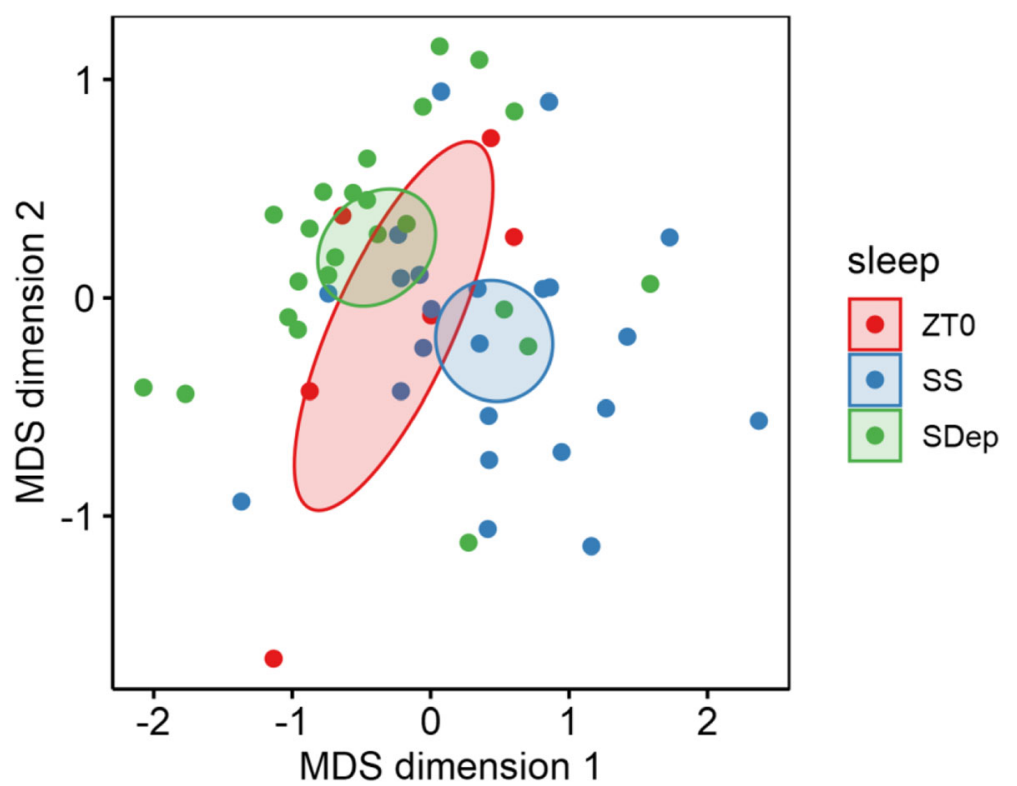

Fig. 2 Plot of the multidimensional scaling (MDS) result. SS and SDep samples formed separate clusters separated primarily on the first dimension, demonstrating that behavioral state explains the largest proportion of gene expression variability. Mice collected at ZTO were between the SS and SDep. Results are shown with confidence ellipse at 95\% confidence intervals

Comparison to the genes differentially regulated between sleep and sleep deprivation in bulk tissue from mPFC To gain further understanding of the sleep and wake regulation in VLPO-Gal neurons and explore differences from other brain regions, we utilized results from data collected from mPFC in a previous RNA-seq study using the same experimental design (i.e. SS vs. SDep over four time-points) [20]. Despite a number of dissimilarities between the two datasets, including brain regions (VLPO vs. mPFC), cell types (enriched galanin neuron vs. bulk tissue), and tissue preparation (micro-punch vs. LCM), 13,068 genes were detected in both studies, corresponding to $85 \%$ of the total detected genes. As expected, Gal and galanin receptor 1 (Galr1) are among the 788 genes uniquely expressed in VLPO-Gal neurons, and cholinergic receptors (Chrna1-5) and microglia-specific genes

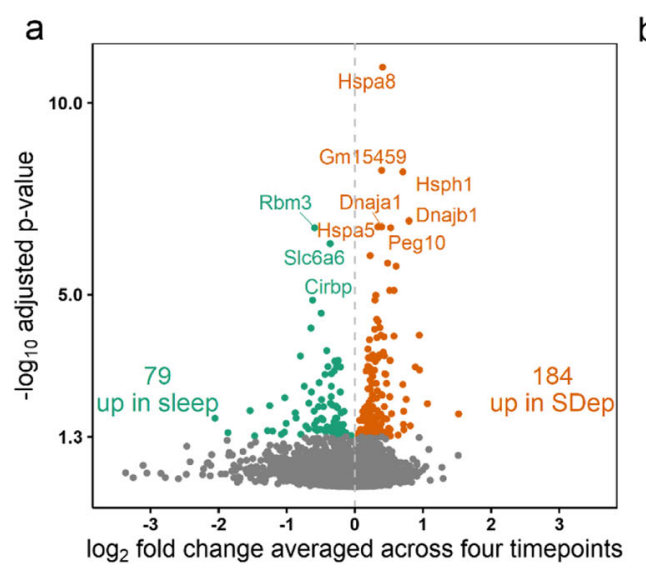

b

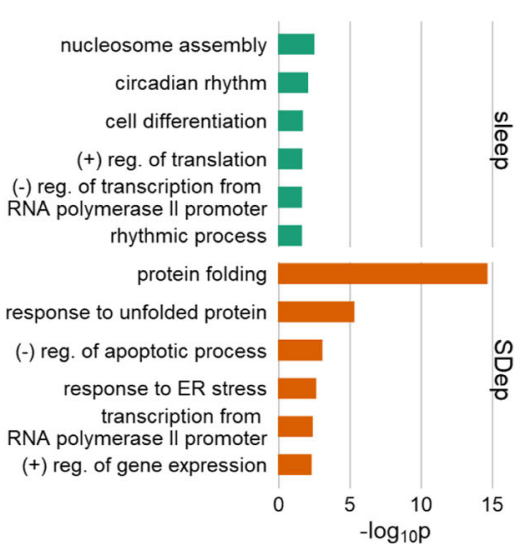

Fig. 3 Differentially expressed genes between spontaneous sleep (SS) and sleep deprivation (SDep). a 184 genes were identified to be significantly up-regulated by SDep (orange) and 79 genes were identified to be significantly up-regulated by sleep (green) with a cutoff of FDR < 0.05. b Selected Biological Processes GO terms enriched in the differentially expressed genes. Protein folding, response to unfolded protein, and regulation of gene expression are among the functions enriched from the genes up-regulated in SDep (red), whereas nucleosome assembly and regulation of translation are among the functions enriched from the genes up-regulated in sleep (green). X-axis shows the negative log 10 of the $P$-values of the $\mathrm{GO}$ enrichment 
Table 1 Correlation with time during SS or SDep among genes differentially regulated between SS and SDep

\begin{tabular}{|c|c|c|}
\hline \multicolumn{3}{|l|}{ Correlation with time during spontaneous sleep (SS) } \\
\hline Genes & $\begin{array}{l}\text { Correlation } \\
\text { with time }\end{array}$ & $\begin{array}{l}\text { Affected by SS (vs. } \\
\text { SDep) }\end{array}$ \\
\hline Dbp, Gpcpd1, Rbm3 & positive & $\begin{array}{l}\uparrow \text { up-regulated with } \\
\text { SS }\end{array}$ \\
\hline Zbtb16, Fkbp5 & positive & $\begin{array}{l}\downarrow \text { down-regulated } \\
\text { with SS }\end{array}$ \\
\hline Stip1, Cct7 & negative & $\begin{array}{l}\downarrow \text { down-regulated } \\
\text { with SS }\end{array}$ \\
\hline \multicolumn{3}{|l|}{ Correlation with time during sleep deprivation (SDep) } \\
\hline Genes & $\begin{array}{l}\text { Correlation } \\
\text { with time }\end{array}$ & $\begin{array}{l}\text { Affected by SDep (vs. } \\
\text { SS) }\end{array}$ \\
\hline $\begin{array}{l}\text { Tsc22d3, Zbtb16, Fkbp5, Lifr, Irs2, Ankrd50, Dnajb5, Hr, Reps2, Srxn1, Mfsd2a, Itga3, Pdia6, Setd7, Mc4r, } \\
\text { Amigo2, Dpp9, Gtf2i, Uba6, Wnt2b, Sfpq, Zbtb40, Calr, 0610009O20Rik }\end{array}$ & positive & $\begin{array}{l}\uparrow \text { up-regulated with } \\
\text { SDep }\end{array}$ \\
\hline Dbp & positive & $\begin{array}{l}\downarrow \text { down-regulated } \\
\text { with SDep }\end{array}$ \\
\hline Nkx6-2, Slc6a6, Ddrgk1, Sox9, Rbm3, 2310022B05Rik, BC067074, Nckap5, Cirbp, Bzrap1, Naaa, Heyl & negative & $\begin{array}{l}\downarrow \text { down-regulated } \\
\text { with SDep }\end{array}$ \\
\hline
\end{tabular}

involved in immune systems, such as C-type lectin family members (Clec5a and Clec4a2), are among the 1588 genes uniquely expressed in mPFC samples.

Among the 13,068 genes commonly detected between the two studies, 254 genes (181 genes up-regulated by SDep and 73 genes up-regulated by SS) were significantly differentially regulated between sleep and SDep in VLPOGal neurons. Surprisingly, large proportions of these genes were also differentially regulated by SS or SDep in the same direction in $\mathrm{MPFC}$, a region that has increased neuronal activity during wake. Specifically, 127 (70.0\%) genes were commonly up-regulated by SDep in both VLPO-Gal and $\mathrm{mPFC}$ and $40(54.8 \%)$ genes were found commonly up-regulated by sleep in both VLPO-Gal and mPFC (Fig. 4 and Additional file 4). As depicted in the heatmap of the fold-changes between SDep and SS across the four timepoints from both studies (Fig. 5a), genes strongly upregulated by SDep in both VLPO-Gal neurons and MPFC are involved in protein folding/ER stress and transcription regulation, including multiple heat shock genes ( $H$ spa 8 and Dnajb1) and transcription factors (Egr1 and Fosl2). Genes up-regulated by SS in both VLPO-Gal neurons and $\mathrm{mPFC}$ are involved in regulation of translation (cold-inducible RNA-binding proteins $R b m 3$ and Cirbp), cell differentiation (Gli1, Sox9, and Spata24), beta-alanine transport (Slc6a6), and circadian rhythm (Dbp).

On the other hand, a subset of genes were regulated in opposite directions by SS and SDep between VLPO-Gal neurons and mPFC. Among the 54 genes up-regulated by SDep in VLPO-Gal neurons only, twelve were activated during SS in mPFC (Fig. 5b). These genes are involved in DNA repair (Herc2 and Mlh3), nervous system development (Epha4, Oprk1, and Srrm4), cellular response to cAMP (Akap9 and Jun), and neuroprotective signaling (Lifr). Similarly, among the 33 genes upregulated by SS only in VLPO-Gal neurons, four genes (Mnt, Cry2, Lrrc23, and Igsf11) were activated by SDep in mPFC (Fig. 5b). Max-binding protein (Mnt) is involved in regulation of transcription and cell cycle, and cryptochrome 2 (Cry2) is critical to glucose homeostasis and other functions related to circadian clock. Therefore, multiple mechanisms exist controlling transcription between SS and SDep in VLPO-Gal neurons. Some of these mechanisms may be systemic (e.g., due to neurohomornal effects or secondary to temperature change), while others could be region specific.

We next examined the Fos expression in VLPO-Gal neurons and mPFC. In VLPO-Gal neurons, Fos was not significantly differential between SS and SDep when compared across the four time points $(F D R=0.19)$. Therefore we compared the Fos levels at individual time-point between SS and SDep. Fos was expressed at a higher level with SDep with nominal significance when compared to the time-matched SS controls at later time points, ZT9 $(p=0.025)$ and ZT12 $(p=0.042)$ (Fig. 6a). The difference observed at these time points was driven by decreased Fos expression with increasing duration of SS toward the end of the light-phase (Pearson's correlation coefficient with number of hours into sleep =$0.40, p=0.055$; Fig. $6 \mathrm{~b}$ ), when lower sleep pressure is expected [12]. In contrast, in MPFC, expression of Fos was highly up-regulated by SDep across the four time-points $\left(\mathrm{FDR}=4.94 \times 10^{-20}\right)$ as well as at all four individual time points (Fig. 6a). Fos showed a trend of increase with duration of SS toward the end of the light-phase in mPFC (Pearson's correlation coefficient $=0.25, p=0.23$ ), indicating that the trend of Fos expression decrease with duration of sleep is specific to the VLPO-Gal neurons. 


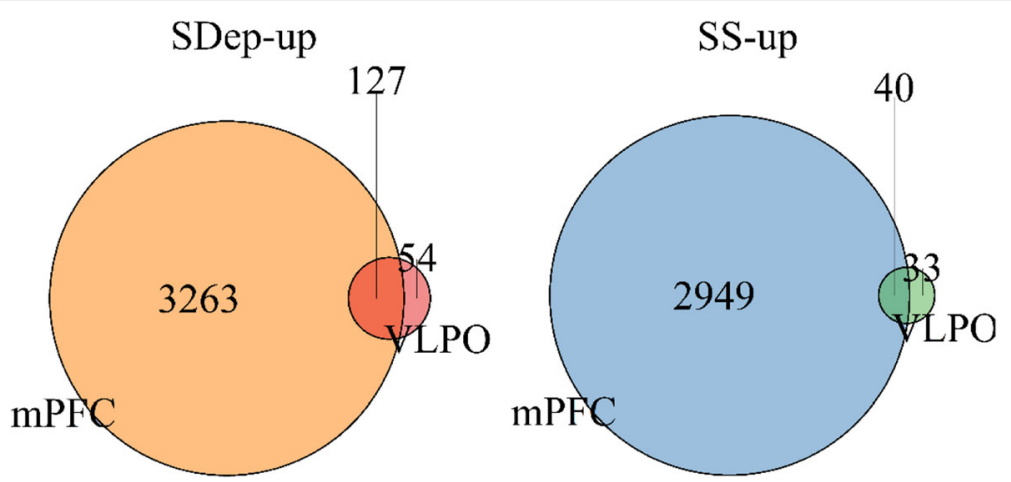

Fig. 4 Venn Diagrams comparing DEGs identified in VLPO-Gal neurons and mPFC. The Venn diagram on the left shows 127 genes are commonly up-regulated by SDep in both VLPO-Gal neurons and mPFC. The Venn diagram on the right shows 40 genes are commonly up-regulated by SS in both VLPO-Gal neurons and MPFC. Specifically in VLPO-Gal neurons, 54 genes are up-regulated by SDep (left) and 33 genes are up-regulated by SS (right)

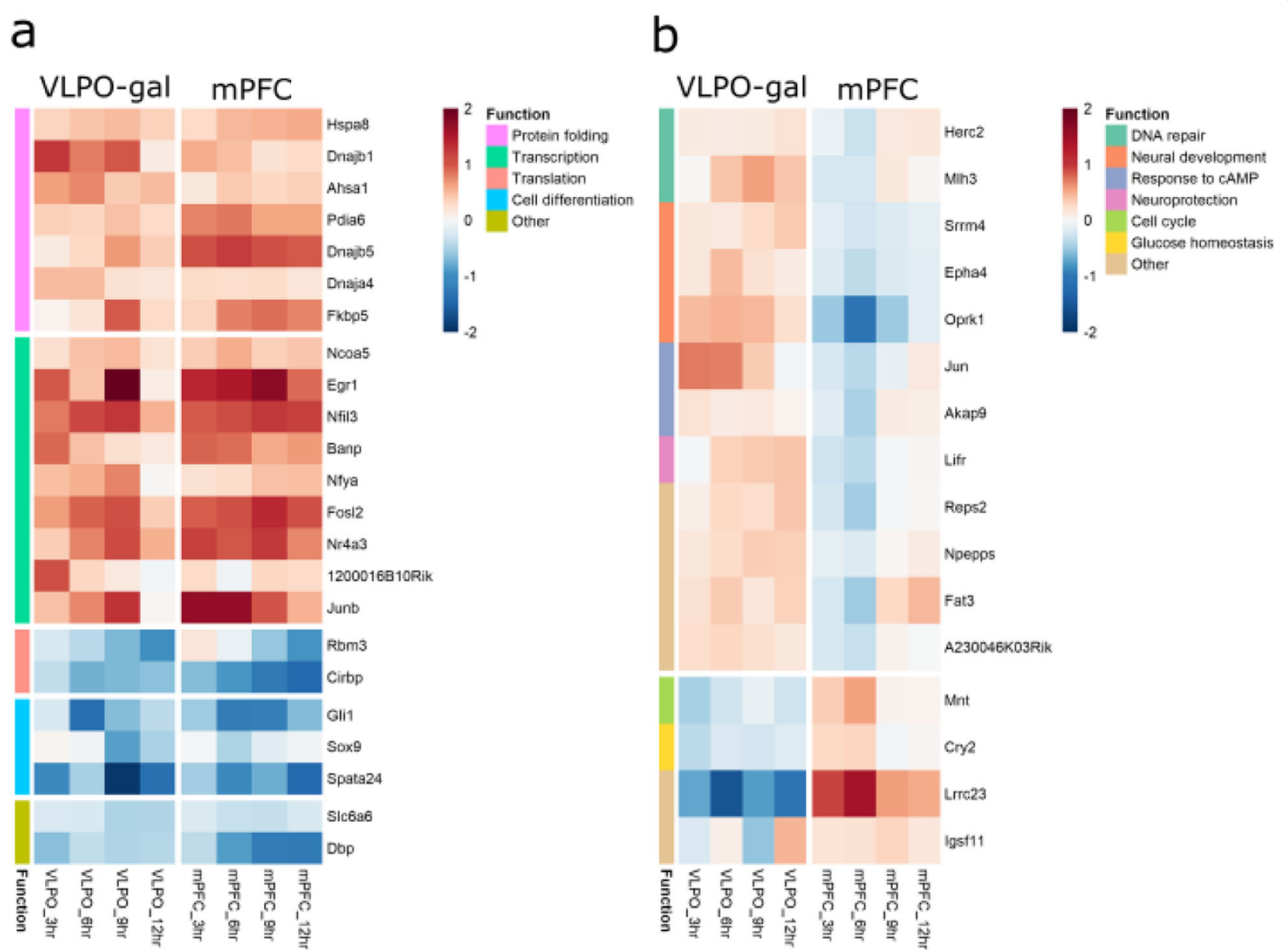

Fig. 5 Heat map of fold-changes between sleep deprivation and sleep at each time point in VLPO-Gal neurons and mPFC. Cell colors with red indicates up-regulation with SDep and blue indicates up-regulation with sleep. Row annotations indicate biological functions. a Genes involved in protein folding and transcription are commonly up-regulated with SDep in both VLPO-Gal and MPFC, whereas translation and cell differentiation are commonly up-regulated with sleep in both VLPO-Gal and mPFC. $\mathbf{b}$ Sixteen genes show opposite direction of change between VLPO-Gal and mPFC cells. Twelve genes were up-regulated with SDep in VLPO-Gal neurons but up-regulated with sleep in mPFC. Functions played by these genes include DNA repair and neuronal development. Four genes (Mnt, Cry2, Lrrc23, and Igsf11) were up-regulated with sleep in VLPO-Gal but upregulated with SDep in MPFC 

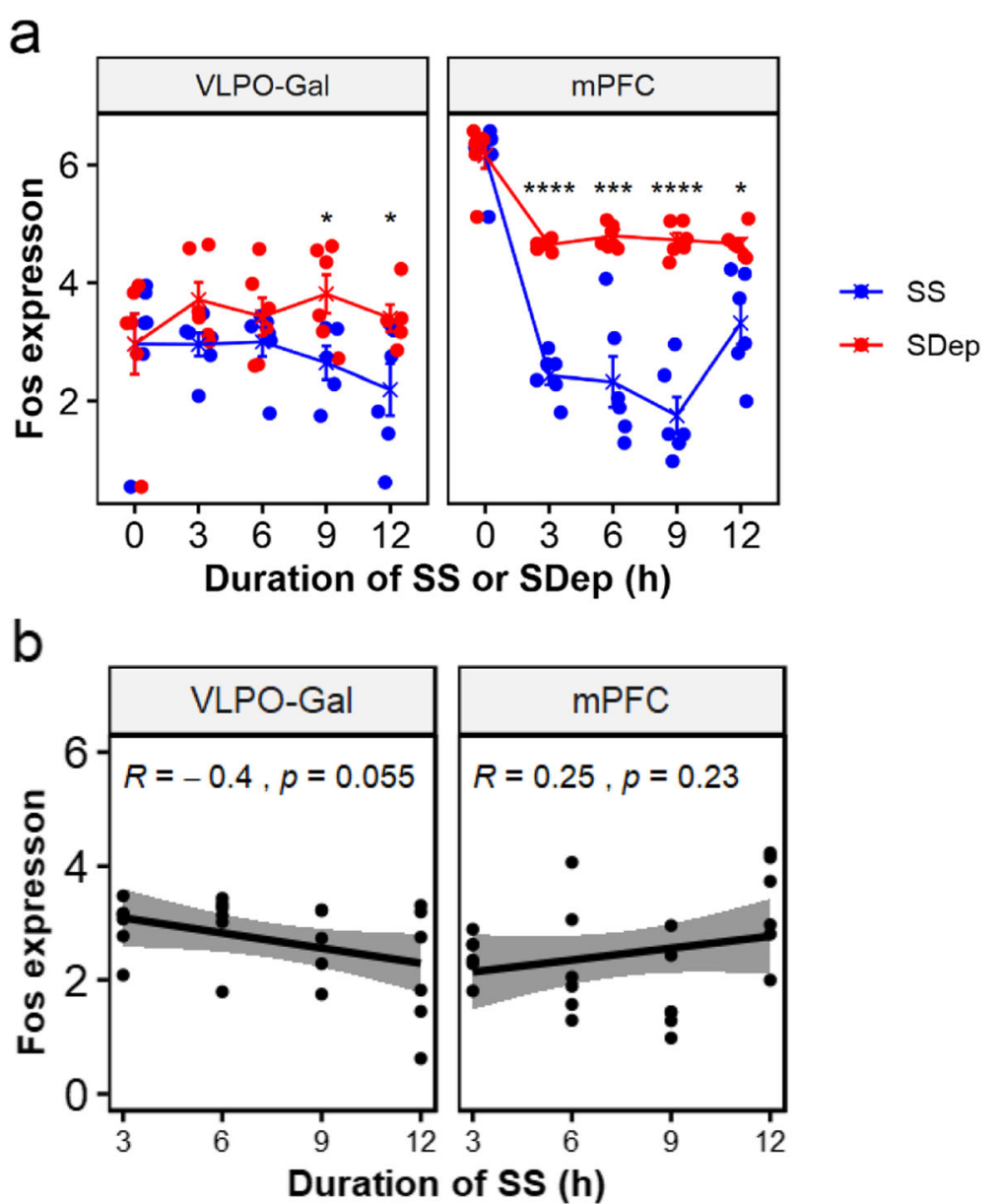

Fig. 6 Expression of Fos across multiple time points during SS or SDep in VLPO-Gal neurons and mPFC. a Expression differences of Fos between SS and SDep. In VLPO-Gal, Fos was not significantly different between SS and SDep at earlier time points ZT3 and ZT6, but expressed at a higher level during SDep at ZT9 and ZT12 when compared using a two sample T-test. In MPFC, Fos was significantly elevated by SDep at all four time points. b Pearson's correlation of Fos expression [log2(CPM + 0.5)] with duration of SS in VLPO-Gal (left) or mPFC (right). In VLPO-Gal, Fos showed a moderately large negative correlation with duration of SS $(R=-0.40, p=0.055)$, although not statistically significant. In mPFC, Fos was not significantly correlated with duration of SS but the direction of change was opposite from that of VLPO-Gal $(R=0.25, p=0.23)$

We then examined the correlation between Fos expression and the amount of sleep in the last hour before sacrifice in VLPO-Gal neurons and mPFC. We observed a trend of positive correlation between the Fos expression in VLPO-Gal neurons and amount of sleep (minutes) in the last hour before sacrifice (Pearson's correlation coefficient $=0.33, p=0.120$; Fig. 7 ). While not statistically significant in our small sample, the observed correlation coefficient is moderately large, based on guidelines provided by Cohen for small $(r h o=0.1)$, moderate $(r h o=$ $0.3)$ and large $(r h o=0.5)$ correlations [11]. Thus, results suggest a moderate association between increased levels of Fos and greater sleep amounts in VLPO-Gal neurons. This correlation was found despite the limited range in sleep amounts among mice in our SS groups (72-97\%; Fig. 7), which were required to have $\geq 70 \%$ in the last hour prior to sacrifice (see Methods). In contrast, in
mPFC, Fos showed a trend of negative correlation with moderate association with the amount of sleep in the last hour before sacrifice (Pearson's correlation coefficient $=-0.38, p=0.063$; Fig. 7). Taken together, these observations suggest that Fos shows region/cell-type specific trends in VLPO-Gal neurons with positive association with the sleep amounts before sacrifice and negative association with prolonged sleep or sleep need.

VLPO-gal samples show significant enrichment of gene sets expressed in galanin-enriched inhibitory neurons clusters identified in a single-cell RNA-seq study

Although there is considerable evidence indicating that galanin cells in VLPO are sleep-active and sleeppromoting $[17,28,49,50]$, a recent study suggests that there might be a mixture of sleep-active and wake-active galanin neurons in VLPO [9]. To assess cellular 


\section{Fos correlation with amount of sleep}

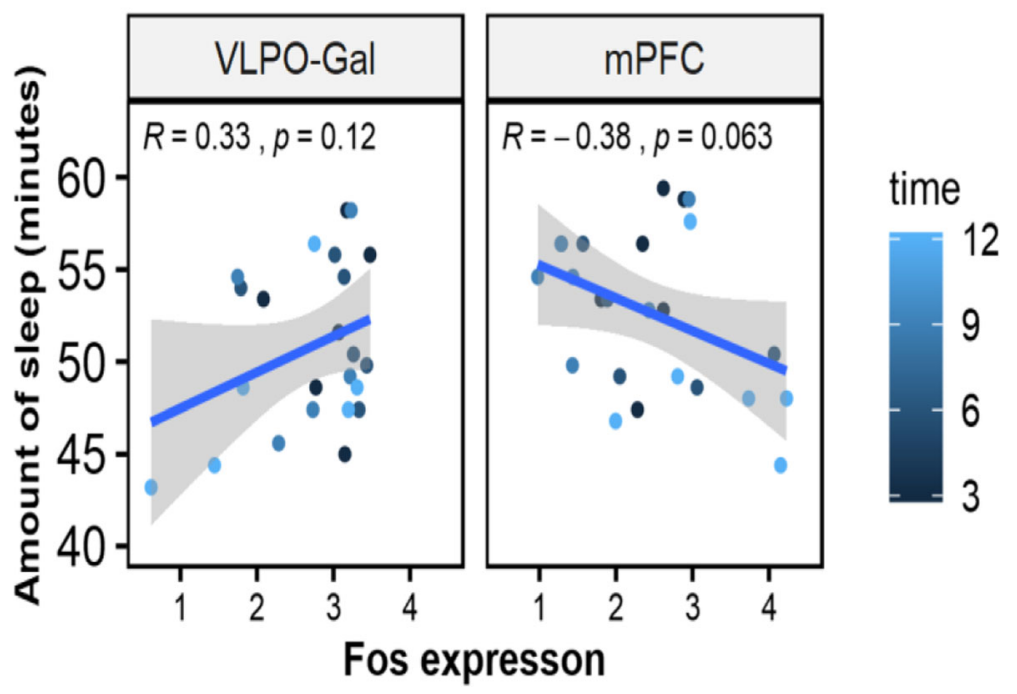

Fig. 7 Pearson's correlation of Fos with the amount (minutes) of sleep in the last $1 \mathrm{~h}$ before sacrifice in VLPO-Gal neurons and $m P F C$. Fos expression $[\log 2(C P M+0.5)]$ in VLPO-Gal had a moderate positive correlation $(R=0.33, p=0.12)$ with the amount of sleep in the last hour before sacrifice, although not statistically significant. Fos expression in MPFC showed opposite direction of change as that of VLPO-Gal $(R=-0.38, p=0.063)$

heterogeneity, we utilized data from a recent single-cell RNA-seq study performed in the preoptic area of hypothalamus [38]. A number of galanin-enriched inhibitory $(n=5)$ and excitatory $(n=3)$ neuron clusters were identified. Among them, three inhibitory clusters (i8, i16, i18) and one excitatory cluster (e3) were localized at or near VLPO using multiplexed error-robust FISH (MERF ISH). Genes highly expressed in these clusters were selected as gene sets (see Additional file 5 and Methods) and tested for enrichment in our LCM isolated VLPOGal samples. Two other inhibitory cell clusters were also considered: i2 [possibly containing the Tac1/Pdyn expressing sleep-active neurons identified by Chung et al. [9]] and i5 (enriched with Pou3f3 instead of galanin near VLPO). The gene sets informed by the galaninexpressing inhibitory neuron clusters i8 and i18 were the most significantly enriched in our VLPO-Gal samples $\left(\mathrm{q}=1.9 \times 10^{-5}\right.$ and $7.9 \times 10^{-5}$, respectively; Table 2$)$, whereas gene sets based on the galanin-expressing excitatory neuron cluster e3 and the Tac1/Pdyn-expressing inhibitory neuron cluster i2 were not enriched in our VLPO-Gal samples ( $q=0.15$ and 0.052) (Table 2). As a sensitivity analysis to ensure that the enrichment results were not dependent on the specific gene set selection criteria or only limited to the chosen cell clusters, we tested enrichment of all 66 cell clusters identified in the single-cell RNA-seq dataset with varying criteria for gene selection (see Methods). The galanin-expressing inhibitory neuron cluster i8 was the most enriched cell cluster on average across sensitivity analyses, and the cell cluster i18 was mostly within the top 5 most enriched cell clusters and consistently more enriched than all other excitatory cell clusters or i2 and i5 (Additional file 5). These results support that our LCM isolated samples were enriched with galanin-expressing inhibitory neurons at or near VLPO, but not with galanin-expressing excitatory neurons or Tac1/Pdyn expressing inhibitory neurons primarily found outside of VLPO. These results, together with the positive association of Fos expression with the amount of sleep, suggest that our VLPO-Gal samples were primarily made up of sleep-active inhibitory neurons.

\section{Discussion}

A total of 263 genes were differentially expressed between SS and SDep in VLPO-Gal neurons. Pathways related to protein folding/ER stress and regulation of transcription were enriched among the 184 genes upregulated during SDep, whereas nucleosome assembly and regulation of translation were functions enriched among the 79 genes up-regulated during SS. A recent single-cell RNA-seq study of the preoptic area identified a number of galanin-enriched neuron clusters situated at or near VLPO [38]. Our gene set enrichment analysis demonstrated that our VLPO-Gal samples were highly enriched with genes expressed in inhibitory neuron cluster i8 (close to the core of VLPO), but not enriched with excitatory neuron cluster e3 (overlap with VLPO) nor the Tac1/Pdyn expressing inhibitory neuron cluster i2 [outside of VLPO, which is possibly the sleep-active neurons identified by Chung et al. [9]]. These results 
Table 2 Gene set enrichment analysis of gal-expressing neuron clusters identified in a single-cell RNA-seq study of preoptic area of hypothalamus [38]

\begin{tabular}{lllll}
\hline Neuron Cluster & Description & Number of Genes & $\boldsymbol{p}$-value & FDR \\
\hline i8 & Gal-inhibitory & 25 & $3.17 \times 10^{-6}$ & $2.64 \times 10^{-4}$ \\
i18 & Gal-inhibitory & 27 & $1.75 \times 10^{-3}$ & $7.92 \times 10^{-4}$ \\
i5 & Pou3f3-inhibitory & 39 & $7.78 \times 10^{-3}$ & $3.50 \times 10^{-3}$ \\
i16 & Gal-inhibitory & 31 & $4.38 \times 10^{-2}$ & $1.17 \times 10^{-2}$ \\
i2 & Tac1/Pdyn-inhibitory & 54 & $1.52 \times 10^{-1}$ & $5.25 \times 10^{-2}$ \\
e3 & Gal-excitatory & 30 & $1.52 \times 10^{-1}$ \\
\hline
\end{tabular}

Neuron cluster gene sets were adapted from [38] using criteria as described in Methods. Description indicates the marker gene enriched in the cluster and if the neuron cluster is inhibitory (starts with " $\mathrm{i}^{\prime \prime}$ ) or excitatory (starts with "e") (for detailed identities of the genes, see Additional file 5). $P$-value and FDR values are twotailed $p$-value and Benjamini-Hochberg controlled FDR values obtained from the camera results, respectively

indicate that our LCM samples were enriched with galanin-expressing inhibitory neurons, but not the galanin-expressing excitatory neurons in VLPO. Earlier studies based on electrophysiological recording and cFos immunochemistry have shown that a majority of the sleep active neurons in VLPO are GABAergic and that the number of c-Fos positive GABAergic neurons correlates positively with the amount of sleep $[16,17]$. In the current study, although not statistically significant, Fos expression showed a moderately large positive correlation [11] with the amount of sleep the animals experienced during the $1 \mathrm{~h}$ before sacrifice. These complimentary analyses indicate that our VLPO-Gal samples contained primarily sleep-active inhibitory neurons.

Genes involved in protein folding and ER stress pathways (Hspa8, Hsph1, and Hspa5) were significantly upregulated by SDep in VLPO-Gal neurons. Previous studies from us and others have demonstrated that these same genes/pathways are consistently up-regulated by wakefulness across multiple brain regions [cortex \& hypothalamus [34], hippocampus [57], mPFC [20], and in wake-active Chat neurons of basal forebrain [42]], as well as in multiple peripheral tissues [liver [36], lung \& heart [2], and pancreas [41]]. This confirms our previous hypothesis that a systemic mechanism is involved in the activation of protein-folding genes in response to extended wakefulness [42]. The activation of protein-folding genes is not specific to SDep or related to glucocorticoid signaling. Results from Cirelli et al. demonstrated the same heat-shock genes were also elevated during spontaneous wakefulness [10]. Similarly, heat shock genes were upregulated in sleep deprived adrenalectomized mice in which glucocorticoid signaling was maintained constant [39]. One possible mechanism for this molecular response is suggested by studies in C. elegans [55] that examined the effect of increasing expression of the spliced form of XBP-1 (XBP-1 s) in neurons. XBP-1 is one component of the unfolded protein response (UPR). XBP-1 $\mathrm{s}$ is a transcription factor that regulates expression of a number of genes involved in ER proteostasis $[1,48]$. Transgenic expression of XBP-1 s in neurons in worms results in a cell-non-autonomous response in which the UPR is activated in multiple tissues [55]. While the basis of this effect was not fully identified, the mechanism involved neurotransmitter release from small vesicles, since the cell-non-autonomous UPR induction was attenuated in worms lacking UNC-13, which have a deficiency of release from small neuronal vesicles. The investigators did not, however, identify the actual signaling molecule [55].

Another set of genes that showed highly consistent changes between VLPO-Gal neurons and other brain regions were cold-inducible RNA-binding proteins (Cirbp and $R b m 3$ ). These genes were also elevated during sleep in peripheral tissues [2]. It has been speculated that expressions of these genes are controlled by the small drop in body temperature during sleep [42]. The change in body temperature across the day in mouse is largely caused by differences in behavioral state (sleep/wake) [21]. Another possible explanation of the systemic regulation of Cirbp across multiple brain regions and cell-types is its important function in circadian gene expressions [40]. A recent study demonstrated that Cirbp altered the changes in clock-gene expression produced by SDep [21]. Moreover, the amount of REM sleep during recovery following SDep is attenuated in Cirbp knockout mice. Cirbp is at the core of the interaction between circadian gene expression and sleep homeostasis.

Interestingly, a subset of genes were regulated between sleep and wake in the opposite direction in VLPO-Gal when compared to our previously published RNA-seq analysis of mPFC [20]. These genes included two involved in DNA repair, Herc2 and Mlh3, which were elevated during SDep in VLPO-Gal samples, but activated during sleep in $\mathrm{mPFC}$. This suggests that the expression of these genes is increased during relative quiescence of the relevant neurons (e.g., during sleep in $\mathrm{mPFC}$ and wake in galanin cells in VLPO). Herc2 is an important mediator of DNA damage response and is critical for the ubiquitin-dependent retention of the DNA repair factors on damaged chromosomes [3]. Mlh3 is important for DNA mismatch repairs [33]. Both genes have been implicated in cancer and 
neurodegenerative diseases $[6,26,43,63]$, indicating their essential roles in maintaining DNA integrity. Recent studies have established direct relationships between neuronal activity and DNA breaks, and DNA breaks in-turn facilitate the induction of immediate early genes [35]. Another study used time-lapse imaging of chromosomal markers of live zebrafish and revealed accumulation of DNA breaks during wakefulness and increased chromosome dynamics (essential for DNA damage repair) during sleep [61]. This is consistent with our finding of elevated DNA repair pathways during sleep in mPFC. In contrast, VLPO-Gal neurons show increased activity during sleep, and reduced activity during wakefulness $[17,50]$. This is likely the reason why we observed increased DNA repair during wakefulness in VLPO-Gal neurons.

By comparing the genes differentially regulated between sleep and wake in MPFC to those in VLPO-Gal neurons, there was a large reduction in the extent of transcriptional regulation in VLPO-Gal cells. Among the 13,068 genes commonly detected between the two studies, 6179 (47.3\%) had significantly altered expressions between SS and SDep in mPFC, whereas only 254 genes (1.9\%) were differentially regulated in VLPO-Gal cells at the same FDR cutoff of 5\%. Similarly, in our previous microarray studies, 3988 and 823 genes were identified to be differentially regulated between sleep and wake in cortex and hypothalamus bulk tissues, respectively [34]. A previous study of Chat neurons in basal forebrain also revealed a small number of genes $(n=10)$ meeting an FDR cutoff of 5\% [42]. These results are consistent with the findings from an in-situ hybridization whole-brain mapping of the effect of SDep [56], which demonstrated that hypothalamus and brainstem are relatively less responsive at the transcriptional level compared to cortex and hippocampus.

Our study is the first to investigate the transcriptional changes between sleep and wake in galanin neurons from VLPO, a region/cell-type that plays important roles in sleep regulation. Although we cannot completely eliminate the possibility of cross-contamination from other cell-types, such as glia cells, our LCM isolated samples showed $>100$-fold enrichment of galanin and $>$ 70-fold depletion of Aldh1l1, a marker of the astrocyte. Therefore, we are confident to conclude that the changes observed are primarily coming from the galanin-expressing neurons. However, there are several limitations that merit discussion. A technical limitation is the use of LCM to isolate GFP-labeled cells requiring fixation using formaldehyde, which has the potential to cause RNA damage [19]. Since the effect of formaldehyde on RNA integrity is temperature and time dependent $[13,31,58]$, we performed post-fix on ice for only $5 \mathrm{~min}$, which minimizes the influence of formaldehyde. For example, one study has shown that the quality of RNA derived from white blood cells incubation with formaldehyde is stable for as long as 3 days at $4{ }^{\circ} \mathrm{C}$ [31]. In addition, we used an optimized RNA extraction buffer for fixed tissues that contains proteinase $\mathrm{K}$ and SDS and obtained good quality RNA samples (mean RIN value of 8.21). However, despite these approaches, formaldehyde may still cause RNA modifications and variations in the captured RNA reads [24]. Therefore, in comparisons between our current data and previously acquired RNAseq data from fresh-frozen mPFC mouse tissues, rather than directly comparing the RNA reads, we restricted the comparisons to the differentially expressed genes identified from the two studies. Another technical challenge of the study is the heterogeneous nature of the hypothalamus. A single-cell RNA-seq study of hypothalamus identified 34 neuronal clusters [8]. Another singlecell RNA-seq study of the preoptic area of hypothalamus identified 43 inhibitory neuron clusters and 23 excitatory neuron clusters [38]. This highlights the need to study cell-type specific gene expression changes in highly heterogeneous regions; our LCM study serves as an initial approach in this direction. However, while more uniform than bulk tissue, a limitation in our data is that the results are still obtained from pooling of hundreds of cells. A future single-cell RNA-seq study is required to further decipher the transcriptomic changes responding to sleep and wake at the single cell level. Finally, a limitation of our study design is the lack of a condition that can dissociate homeostatic sleep pressure from wakefulness. Fos showed a trend of decreasing with longer time spent asleep toward the end of the light phase, a time where Fos was also expressed at higher levels under SDep compared to time-matched SS controls. These observations agree with the previous findings that the activities of VLPO-Gal neurons correlate to sleep need and increase under sleep pressure induced by SDep $[17,50]$. However, since SDep increases both sleep pressure and wakefulness, the current study is not designed to specifically confirm this conclusion, as we cannot exclude the possibility that VLPO-Gal neurons are activated by wakefulness or by both sleep pressure and wakefulness. A future study should also examine expression changes of VLPO-Gal neurons under recovery sleep after SDep to investigate the transcriptional regulation involved in homeostatic sleep control.

\section{Conclusions}

In conclusion, our results indicate that at the transcriptional level both wake-active and sleep-active neurons show increased expression of genes in the endoplasmic reticulum unfolded protein response during wakefulness, while during sleep they show increased expression of genes for cold-induced RNA binding proteins. Thus, these effects are systemic and indeed found in organs 
other than brain $[2,36,41]$. In contrast, we found that expression of DNA repair genes is increased in VLPOGal cells during wakefulness, but increased in wakeactive cells during sleep $[4,20]$. The increase in expression of these genes during the cell-type-specific quiescent periods suggests expression may be driven by region or cell-type specific activity - DNA breaks occur when neurons are active and are repaired when neurons are relatively inactive. The role of these changes in regulation of sleep and wake remains to be determined.

\section{Methods}

\section{Mouse experiments}

Male Tg (Gal-EGFP)HX109Gsat mice on a SwissWebster background that express eGFP under the control of galanin promoter were obtained from the Mutant Mouse Resource \& Research Centers. Mice at 2-3 months of age were housed individually in a pathogenfree, temperature $\left(22^{\circ} \mathrm{C}\right)$, and humidity $(45-55 \%)$ controlled room with a 12-h/12-h light/dark cycle with lights-on at 7 AM (Zeitgeber time zero or ZT0). Water and food were available ad libitum. Animals were acclimated for 14 days to individual housing and the presence of experimenter prior to the experiment. There were two groups of mice-one that was sleep deprived by gentle handling and one that was allowed to sleep spontaneously. On the days of the experiments, SDep was initiated using gentle handling [14] at lights-on, and mice were collected following 3, 6, 9, and $12 \mathrm{~h}$ of SDep ( $n=5-6$ at each time point). For mice that were allowed SS at matching time-points ( $n=5-6$ at each time point), sleep was monitored starting from lights-on; only mice that slept for $\geq 70 \%$ of the last $1 \mathrm{~h}$ before sacrifice were included in the SS group. Sleep was monitored using the AccuScan infrared monitoring system that detects movement when the mouse crosses electronic beams, and based on previous validation studies performed with Swiss-Webster male mice of 3 months of age using EEG/ EMG, sleep was defined as $\geq 50$ s of continuous inactivity [42]. Additional mice $(n=6)$ were also chosen randomly for "baseline" assessment of gene expression and sacrificed at ZT0. In total, 52 mice were used for the study of gene expression differences between SS and SDep. An additional seven animals under undisturbed conditions were collected during the light phase and were used in the study to test for the enrichment of galanin neurons in isolated eGFP $(+)$ cells. Therefore, a total of 59 mice were used in this study.

\section{Tissue collection}

Mice were euthanized by cervical dislocation and brains were quickly removed and rinsed in nuclease-free $1 \mathrm{x}$ phosphate buffered saline (PBS). A $3 \mathrm{~mm}$ coronal section (Bregma +1.5 to -1.5 ) containing the ventrolateral preoptic (VLPO) region was incubated in ice-cold $4 \%$ formaldehyde ( $\mathrm{pH} 7.4$ ) for $5 \mathrm{~min}$, rinsed twice with PBS, frozen in cryo-embedding medium OCT (Tissue-Tek), and stored at $-80^{\circ} \mathrm{C}$ until sectioning. $10 \mu \mathrm{m}$ sections were made in a cryostat kept at $-20^{\circ} \mathrm{C}$. The VLPO region was identified based on the mouse atlas of Paxinos and Franklin's [15]. Using the anterior commissure as a landmark and the eGFP labeled galanin neurons as a guidance, approximately 50 brain slices containing the VLPO region (Bregma +0.40 to -0.10 ) per animal were captured on regular glass microscope slides, $2-3$ sections per slide, and stored in covered slide box and sealed in plastic bags at $-80^{\circ} \mathrm{C}$ until use of LCM.

\section{Laser capture microdissection (LCM)}

LCM was performed as described previously [42]. Briefly, brain sections were dehydrated immediately before using the LCM protocol: air dry (30 s), 75\% ethanol (30 s), RNase-free water (15 s), 75\% EtOH (30 s), 95\% $\mathrm{EtOH}(30 \mathrm{~s}), 1$ st $100 \%$ ethanol (30 s), 2nd 100\% ethanol (1 $\mathrm{min}), 1$ st xylene $(1 \mathrm{~min})$, and 2 nd xylene $(3 \mathrm{~min})$, and finally air dried in hood for 5 min to remove residual xylene. Between each transition from buffer to buffer, the slides were quickly tapped against the tube rim to remove excess liquid. Dehydrated slides were kept in clean and dried microscope slide boxes containing molecular sieves until ready for LCM. Gal-eGFP(+) neurons from VLPO were dissected using Arcturus XT LCM system (MDS analytical technologies). Since the short $5 \mathrm{~min}$ post-fix did not result in deep penetration of formaldehyde, neurons in the dorsal extension of VLPO and the medial and median preoptic nuclei were mostly not visible, whereas GFP fluorescent at the VLPO core and the medial extension were well preserved. GFP-labeled cells were dissected primarily from the VLPO core with care taken to avoid picking galanin neurons from the VLPO medial extension based on the staining of all galanin neurons in POA described elsewhere [28]. The thermoplastic film on the cap was carefully peeled off and immersed in $100 \mu \mathrm{l}$ lysis buffer $(10 \mathrm{mM}$ Tris- $\mathrm{HCl}[\mathrm{pH}$ 7.5], $50 \mathrm{mM}$ EDTA [pH 8.0], $0.2 \mathrm{M} \mathrm{NaCl}, 2.2 \%$ SDS, RNase inhibitor and $1000 \mathrm{mg} / \mathrm{ml}$ proteinase $\mathrm{K}$ ) and incubated at $55^{\circ} \mathrm{C}$ for $30 \mathrm{~min}$ with gentle shaking. This lysis method was adapted based on an optimized method for fixed tissues [25]. After lysis, the tube was stored at $80^{\circ} \mathrm{C}$ until RNA extraction.

\section{RNA purification, sequencing and bioinformatics}

RNA extraction was performed using an RNAqueousMicro Total RNA Isolation Kit (Invitrogen) and oncolumn DNase digestion was performed using an RNase-Free DNase Set (Qiagen) following the manufacturers' protocols. RNA concentration and qualities were accessed by Agilent 2100 Bioanalyzer RNA 6000 Pico 
chip. All samples had RIN values above 7.0, with a mean (standard deviation) RIN of 8.21 (0.52). Library preparation was performed using a SMARTer ${ }^{\circ}$ Stranded Total RNA-Seq Kit v2 - Pico Input Mammalian by Takara Bio, and sequencing done with 150 base-pair paired-end reads on Illumina HiSeq 4000. Raw reads were aligned to the mouse genome build $\mathrm{mm} 9$ by STAR version 2.5.3a, and quantified at the gene level using scripts from the PORT pipeline (github.com/itmat/Normalization -v0.8.4-beta). Low-expressing genes were removed by keeping the genes with mean of 10 counts or higher across all samples. A total of 13,856 genes with unique ensemble IDs that passed this filtering criteria were normalized using the "Trimmed Mean of M-values" (TMM) method in the edgeR package [45], and differential expression analyses were performed using the LimmaVoom package [44] (see also Differential gene expression and functional analysis, below).

\section{Test enrichment of galanin in eGFP(+) samples}

eGFP(+) neurons from VLPO core were collected using LCM from four animals collected during undisturbed conditions during light phase as described above following the same procedures. eGFP(-) cells were collected from the adjacent piriform cortex region on the same sections in the same animals using LCM. VLPO bulk tissue was collected from the remaining three mice, which were euthanized by cervical dislocation and whole brains were quickly removed and frozen on dry ice. VLPO region bulk tissue (Bregma +0.4 to -0.1 , lateral $\pm 0.5 \mathrm{~mm}$ ) was punched using a $0.7 \mathrm{~mm}$ micropunch based on the mouse atlas of Paxinos and Franklin's [15] in a cryostat and using the anterior commissure as a landmark. This micropunch method has previously been used for our studies of mPFC [20]. Enrichment of galanin expressing neurons and depletion of astrocytes in eGFP $(+)$ samples were tested by comparing expression of Gal and Aldh1l1 in eGFP $(+)$ samples $(n=4)$, eGFP $(-)$ samples $(n=4)$, and the VLPO bulk tissue $(n=3)$. Real time PCR was performed using the following TaqMan assays: Gal (Mm00439056_m1), Aldh1l1 (mm03048957_m1), Tbp (mm00446971_m1), and Hprt (mm01545399_m1). Relative expressions of Gal and Aldh1l1 were calculated using the 2- $\Delta \Delta C T$ method [47] with the reference values being the geometric means of the two housekeeping genes, Tbp and Hprt. Comparisons of Gal and Aldh1l1 expression were made among the LCM samples $[\mathrm{eGFP}(+)$ or eGFP(-)] and the VLPO bulk samples on the $-\Delta \mathrm{Ct}$ level using an exact $p$-value from the KruskalWallis test. To test for enrichment of $\mathrm{Gal}$ in $\operatorname{eGFP}(+)$ samples, two separate pairwise comparisons were made: eGFP(+) vs. eGFP(-) and eGFP(+) vs. VLPO bulk samples, using a one-tailed Wilcoxon exact test, with median and $95 \%$ confidence intervals of differences derived using the Hodges-Lehmann estimate. Similarly, pairwise comparisons of eGFP(+) vs. VLPO bulk and eGFP(-) vs. VLPO bulk were performed to test for depletion of Adlh1l1 in LCM samples compared to the bulk tissue. Analyses were performed using $\mathrm{R}$ (www.r-project.org) and SAS Version 9.4 (SAS Institute, Cary, NC).

\section{Differential gene expression between sleep and sleep deprivation}

Using the LimmaVoom package in R software [30], we evaluated the differentially expressed genes (DEGs) between sleep deprived and spontaneous sleep behavioral states (SDep vs. SS) across all time points. Specifically, SDep samples were compared with their time-matched SS controls at each of the four time durations $(3,6,9$, and 12 h), and a moderated F-test was used to assess a global statistical difference between SS and SDep for each gene combining t-statistics for all contrasts at individual time points [44]. Multiple comparisons correction for statistical significance was applied using the method of Benjamini and Hochberg [5] to control the overall false discovery rate (FDR) at 5\%. A multidimensional scaling (MDS) plot of all samples was generated using the plotMDS function in Limma with default parameters. The Euclidean distance between each pair of samples, calculated as the rootmean-square deviation of the top 500 most variable genes automatically selected for each pair of samples, was used as an indication of the similarity between samples [44]. The first dimension represents the leading-fold-change that best separates samples and explains the largest proportion of variation in the data, with subsequent dimensions having a smaller effect and being orthogonal to the ones before it [30].

\section{Temporal expression changes during sleep and sleep deprivation}

To explore the time-dependent changes in gene expression during sleep and sleep deprivation, Pearson's correlation coefficients were calculated between ordinal time (ZT0, ZT3, ZT6, ZT9, and ZT12) and gene expression values, combining animals $(n=6)$ collected at lights-on as time zero and animals collected during SS and SDep across the four time points (ZT3, 6, 9, and $12)$, separately. Gene expression correlation with time was first tested for the DEGs differentially regulated between SS and SDep, with statistical significance based on an FDR correction following the method of Benjamini and Hochberg [5]. Secondly, as a broader discovery analysis gene expression correlation with time was tested for all detected genes. The correlation coefficients obtained were ranked from highest to lowest and pathway enrichment was explored using the gene set enrichment test via Camera [59] against the MSigDB gene sets for mouse (v5.2) [32]. 


\section{Comparison to RNA-seq data of medial prefrontal cortex (mPFC)}

Data and results were obtained from a previously published RNA-seq study performed on $\mathrm{mPFC}$ using the same experimental design [20]. Differentially regulated genes between SS and SDep in mPFC identified from the young animals (2-4 months) were used to compare with data obtained from this study. The mPFC data are publicly available at Gene Expression Omnibus (GSE128770).

\section{Functional analysis}

Functional gene ontology (GO) analysis was performed using DAVID Bioinformatics Resources 6.8 [22]. Unless otherwise noted, enrichment of GOs was performed against GOTERM_BP_Direct (Biological Process), GOTERM_MF_Direct (Molecular Function), and GOTERM_CC_Direct (Cellular Component). For DEGs identified in VLPO-Gal neurons, the GO enrichment tests were performed for the genes up-regulated by SDep and the genes up-regulated by SS, separately. For DEGs also differentially regulated in the same direction in mPFC, the GO enrichment tests were performed for the common up-regulated genes by SDep and the common up-regulated genes by SS, separately. Enrichment of GO terms was defined as a $p<0.05$ and containing at least 2 genes. The 13,856 genes used for identifying DEGs were used as the background in all cases.

\section{Gene set enrichment analysis}

Gene sets of different neuronal clusters were chosen based on the preoptic area single-cell RNA-seq data by Moffitt et al. [38]. The following neuron clusters were chosen: galanin-enriched inhibitory neurons at or near VLPO (i8, i16, i18), gal-enriched excitatory neurons near VLPO (e3), Tac1/Pdyn-enriched inhibitory neurons in horizontal limb of the diagonal band of Broca (HDB) (i2), and Pou3f3-enriched inhibitory neurons at VLPO (i5). The marker gene set of a given cell cluster was defined as having $10 \%$ higher expression than the mean expression across all the cell clusters and being detectable in $>25 \%$ of all cells in the corresponding cell cluster [7]. The resulted gene sets are shown in Additional file 5. Gene set enrichment was run using Camera from the Limma package using default parameters against a gene list ranked based on mean expression averaged across all VLPO-Gal samples [59]. The gene set enrichment analysis was further tested across 18 different selection criteria to explore if the enrichment results were stable. Selection criteria were chosen as combinations of three possible expression levels $(5,10 \%$, or $20 \%$ higher than mean) and six possible detection levels (> 15, 20, 25, 30, $40 \%$, or $50 \%$ of cells).

\section{Association of Fos expression with sleep}

Previous studies relying on c-Fos immunoreactivity demonstrated increased c-Fos expression in VLPO with greater amounts of sleep [17, 50]. Here, we calculated Pearson's correlations to test for an association between Fos expression and minutes of sleep in the last hour prior to sacrifice. This was done for the studies reported here and from our previous data on mPFC.

\section{Power calculation}

Power calculation for the RNA-seq study was performed using the RnaSeqSampleSize package and its web interface (https://cqs-vumc.shinyapps.io/rnaseqsamplesizeweb/) [62]. Based on our prior data and results in mPFC [20], we expected to detect 14,000 genes, and that up to 5000 may be differentially expressed between conditions. At an FDR of $5 \%$, a total of 48 animals $(n=24$ per condition) would provide $84 \%$ power to detect a minimum fold-change of 2.0 between groups.

\section{Supplementary information}

Supplementary information accompanies this paper at https://doi.org/10. 1186/s12864-020-07050-7.

Additional file 1: Figure S1. Distribution of galanin neurons in VLPO in
post-fix mouse brain. Galanin neurons have been shown to be distributed
in a few different structures besides the VLPO core (VLPOC). These
include the medial (VLPOem) and dorsal (VLPOed) extensions, as well as
the median (MnPO), medial (MPO), and periventricular preoptic (PVPO)
nuclei [28]. A shows a reference galanin neuron distribution at around
Bregma + 0.14, for which the brain was prepared using perfusion with
$10 \%$ formalin. B shows a brain slide at approximately the same location
from a brain sample prepared using 5 min post-fix with $4 \%$ formaldehyde,
the same as the samples used for LCM in this study. C shows the en-
larged image containing the GFP-labeled galanin neurons. The different
structures were drawn based on the reference image. The outlines were
drawn in the absence of Nissl staining and were for illustration only. As
shown in the images (B and C), the short post-fix allowed well penetra-
tion of formalin (fix the GFP protein) primarily in the VLPO-core but not
deep enough to reach the dorsal extension (VLPOed), MPO, MnPO, or the
dorsal part of the PVPO. AC, Anterior commissure; 3 V, 3rd ventricle; OC,
Optic chiasm. Figure S2. Identification and dissociation of eGFP-galanin
neurons from VLPO core using LCM. A shows a representative brain section
at approximately + 0.15 Bregma under fluorescence with the different
structures illustrated based on the reference image shown in Supplemen-
tary figure 1. The outlines were drawn in the absence of Nissl staining
and were for illustration only. B shows the same brain slide after the
eGFP (+)-galanin cells being removed in the VLPO core structure. Care
was taken to avoid selecting other eGFP(+) cells in the surrounding struc-
tures and regions. The purple circles indicate the exact location where
the cells were dissociated. The image is directly taken from the LCM in-
strument. C shows the captured cells on the CapSure HS cap.
Additional file $\mathbf{2}$.
Additional file 3.
Additional file $\mathbf{4}$.
Additional file 5.

\section{Abbreviations}

BP: Biological process; CC: Cellular component; DEGs: Differentially expressed genes; DR: Dorsal raphe; EEG/EMG: Electroencephalogram/electromyography; eGFP: Enhanced green fluorescent protein; ER: Endoplasmic reticulum; FDR: False discovery rate; Gal: Galanin; GO: Gene ontology; HDB: Horizontal 
limb of the diagonal band of Broca; LC: Locus coreleus; LCM: Laser captured microdissection; MERFISH: Multiplexed error-robust fluorescence in situ hybridization; MF: Molecular function; mPFC: Medial prefrontal cortex; NREM: Non-rapid eye movement; POA: Preoptic area; REM: Rapid eye movement; RNA-seq: RNA-sequencing; SDep: Sleep deprivation; SS: Spontaneous sleep; TMN: Tuberomammillary nucleus; VLPO: Ventrolateral preoptic nucleus

\section{Acknowledgements}

We thank Dr. Jonathan Schug for his assistance on the RNA-Seq experiment, May Chen for animal breeding, and Lin Zhang and May Chen for helping with the sleep deprivation experiment. The abstract has been published on Sleep Medicine (Volume 64, Supplement 1, pages S139-S140, 2019, https:// doi.org/10.1016/j.sleep.2019.11.382).

\section{Authors' contributions}

XF.G. performed the LCM and RNA-seq experiments and data analysis, drafted and revised the manuscript. XL.G. performed the LCM experiments. B.T.K assisted data analysis and revised the manuscript. J.Z. assisted the mouse and LCM experiments. D.S. and G.R.G. contributed to data analysis. J.L. and R.J.G. contributed to the mouse experiments. A.I.P. designed the study, assisted in data interpretation, and revised the manuscript. All the authors have read and approved the final version.

\section{Funding}

Research was supported by National Institutes of Health $(\mathrm{NIH})$ grant P01 AG017628. The funding agency had no role in design and conduct of the study or in preparation of the manuscript.

\section{Availability of data and materials}

All raw and processed RNA-seq data generated in this study have been submitted to the NCBI Gene Expression Omnibus (GEO) under accession number GSE132308 (https://www.ncbi.nlm.nih.gov/geo/query/acc.cgi?acc=GSE1323 08). The mPFC data are publicly available at GEO (https://www.ncbi.nlm.nih gov/geo/query/acc.cgi?acc= GSE128770) using the mouse (Mus musculus) genome build NCBI37/mm9 assembly by the Mouse Genome Sequencing Consortium (https://www.ncbi.nlm.nih.gov/assembly/GCF_000001635.18/).

\section{Ethics approval and consent to participate}

The mice used in this study were purchased from Mutant Mouse Resource \& Research Centers. All animal husbandry and procedures were approved by the Institution of Animal Care and Use Committee of the University of Pennsylvania and were carried out in accordance with all National Institutes of Health guidelines.

\section{Consent for publication}

Not applicable.

\section{Competing interests}

The authors declare that they have no competing interests.

\section{Author details}

'Division of Sleep Medicine/Department of Medicine, University of Pennsylvania Perelman School of Medicine, Philadelphia 19104, USA. ${ }^{2}$ Department of Respiratory and Critical Care Medicine, Second Hospital of Shanxi Medical University, Taiyuan 030001, Shanxi, China. ${ }^{3}$ Institute for Translational Medicine and Therapeutics, University of Pennsylvania, Philadelphia 19104, USA. ${ }^{4}$ Present address at National Institute on Aging, National Institutes of Health, Baltimore 21224, USA. ${ }^{5}$ Department of Genetics, University of Pennsylvania, Philadelphia 19104, USA

Received: 8 January 2020 Accepted: 3 September 2020 Published online: 14 September 2020

\section{References}

1. Acosta-Alvear D, Zhou Y, Blais A, Tsikitis M, Lents NH, Arias C, Lennon CJ Kluger $Y$, Dynlacht BD. XBP1 controls diverse cell type- and conditionspecific transcriptional regulatory networks. Mol Cell. 2007;27(1):53-66.

2. Anafi RC, Pellegrino R, Shockley KR, Romer M, Tufik S, Pack Al. Sleep is not just for the brain: transcriptional responses to sleep in peripheral tissues. BMC Genomics. 2013;14:362.
3. Bekker-Jensen S, Rendtlew Danielsen J, Fugger K, Gromova I, Nerstedt A, Lukas C, Bartek J, Lukas J, Mailand N. HERC2 coordinates ubiquitindependent assembly of DNA repair factors on damaged chromosomes. Nat Cell Biol. 2010;12(1):80-6 sup pp 81-12.

4. Bellesi M, Bushey D, Chini M, Tononi G, Cirelli C. Contribution of sleep to the repair of neuronal DNA double-strand breaks: evidence from flies and mice. Sci Rep. 2016;6:36804.

5. Benjamini Y, Hochberg Y. Controlling the false discovery rate - a practical and powerful approach to multiple testing. J Royal Stat Soc Series BMethodol. 1995;57(1):289-300.

6. Bonanno L, Costa C, Majem M, Sanchez JJ, Rodriguez I, Gimenez-Capitan A, Molina-Vila MA, Vergnenegre A, Massuti B, Favaretto A, Rugge M, Pallares C, Taron M, Rosell R. Combinatory effect of BRCA1 and HERC2 expression on outcome in advanced non-small-cell lung cancer. BMC Cancer. 2016;16:312.

7. Cai Y, Dai Y, Wang Y, Yang Q, Guo J, Wei C, Chen W, Huang H, Zhu J, Zhang C, Zheng W, Wen Z, Liu H, Zhang M, Xing S, Jin Q, Feng CG, Chen X. Single-cell transcriptomics of blood reveals a natural killer cell subset depletion in tuberculosis. EBioMedicine. 2020;53:102686.

8. Chen $R$, Wu X, Jiang L, Zhang Y. Single-cell RNA-Seq reveals hypothalamic cell diversity. Cell Rep. 2017;18(13):3227-41.

9. Chung S, Weber F, Zhong P, Tan CL, Nguyen TN, Beier KT, Hormann N, Chang WC, Zhang Z, Do JP, Yao S, Krashes MJ, Tasic B, Cetin A, Zeng H, Knight ZA, Luo L, Dan Y. Identification of preoptic sleep neurons using retrograde labelling and gene profiling. Nature. 2017;545(7655):477-81.

10. Cirelli C, Gutierrez CM, Tononi G. Extensive and divergent effects of sleep and wakefulness on brain gene expression. Neuron. 2004;41(1):35-43.

11. Cohen J. Statistical power analysis for the behavioral sciences. New York: Routledge Academic; 1988.

12. Curie $T$, Mongrain $V$, Dorsaz $S$, Mang GM, Emmenegger $Y$, Franken $P$. Homeostatic and circadian contribution to EEG and molecular state variables of sleep regulation. Sleep. 2013;36(3):311-23.

13. Eisele $\mathrm{S}$, Krumbholz M, Fischer MT, Mohan H, Junker A, Arzberger T, Hohlfeld $R$, Bradl M, Lassmann $H$, Meinl E. Prospects of transcript profiling for mRNAs and MicroRNAs using formalin-fixed and paraffin-embedded dissected autoptic multiple sclerosis lesions. Brain Pathol. 2012;22(5):607-18.

14. Franken P, Dijk DJ, Tobler I, Borbely AA. Sleep-deprivation in rats - effects on Eeg power spectra, vigilance states, and cortical temperature. Am J Physiol. 1991:261(1):R198-208.

15. Franklin K, Paxinos G. The Mouse Brain in Stereotaxic Coordinates, Elsevier (3rd Edition); 2008

16. Gaus SE, Strecker RE, Tate BA, Parker RA, Saper CB. Ventrolateral preoptic nucleus contains sleep-active, galaninergic neurons in multiple mammalian species. Neuroscience. 2002;115(1):285-94.

17. Gong H, McGinty D, Guzman-Marin R, Chew KT, Stewart D, Szymusiak R. Activation of c-fos in GABAergic neurones in the preoptic area during sleep and in response to sleep deprivation. J Physiol. 2004;556(Pt 3):935-46.

18. Gong H, Szymusiak R, King J, Steininger T, McGinty D. Sleep-related c-Fos protein expression in the preoptic hypothalamus: effects of ambient warming. Am J Physiol Regul Integr Comp Physiol. 2000;279(6):R2079-88.

19. Groelz D, Sobin L, Branton P, Compton C, Wyrich R, Rainen L. Non-formalin fixative versus formalin-fixed tissue: a comparison of histology and RNA quality. Exp Mol Pathol. 2013;94(1):188-94.

20. Guo X, Keenan BT, Sarantopoulou D, Lim DC, Lian J, Grant GR, Pack Al. Age attenuates the transcriptional changes that occur with sleep in the medial prefrontal cortex. Aging Cell. 2019;18(6):e13021.

21. Hoekstra MM, Emmenegger Y, Hubbard J, Franken P. Cold-inducible RNAbinding protein (CIRBP) adjusts clock-gene expression and REM-sleep recovery following sleep deprivation. Elife. 2019;8:e43400.

22. Huang DW, Sherman BT, Lempicki RA. Systematic and integrative analysis of large gene lists using DAVID bioinformatics resources. Nat Protoc. 2009;4(1):44-57.

23. John J, Kumar VM. Effect of NMDA lesion of the medial preoptic neurons on sleep and other functions. Sleep. 1998;21(6):587-98.

24. Jones W, Greytak S, Odeh H, Guan P, Powers J, Bavarva J, Moore HM. Deleterious effects of formalin-fixation and delays to fixation on RNA and miRNA-Seq profiles. Sci Rep. 2019;9(1):6980.

25. Khodosevich K, Inta D, Seeburg PH, Monyer H. Gene expression analysis of in vivo fluorescent cells. PLoS One. 2007;2(11):e1151.

26. Korhonen MK, Vuorenmaa E, Nystrom M. The first functional study of MLH3 mutations found in cancer patients. Genes Chromosomes \& Cancer. 2008; 47(9):803-9. 
27. Koyama Y, Hayaishi O. Firing of neurons in the preoptic/anterior hypothalamic areas in rat: its possible involvement in slow wave sleep and paradoxical sleep. Neurosci Res. 1994;19(1):31-8.

28. Kroeger D, Absi G, Gagliardi C, Bandaru SS, Madara JC, Ferrari LL, Arrigoni E, Munzberg H, Scammell TE, Saper CB, Vetrivelan R. Galanin neurons in the ventrolateral preoptic area promote sleep and heat loss in mice. Nat Commun. 2018;9(1):4129

29. Landry M, Roche D, Angelova E, Calas A. Expression of galanin in hypothalamic magnocellular neurones of lactating rats: co-existence with vasopressin and oxytocin. J Endocrinol. 1997;155(3):467-81.

30. Law CW, Alhamdoosh M, Su S, Dong X, Tian L, Smyth GK, Ritchie ME. RNA seq analysis is easy as 1-2-3 with limma, Glimma and edgeR. F1000Res. 2016;5:1408

31. Letzkus M, Luesink E, Starck-Schwertz S, Bigaud M, Mirza F, Hartmann N, Gerstmayer B, Janssen U, Scherer A, Schumacher MM, Verles A, Vitaliti A, Nirmala N, Johnson KJ, Staedtler F. Gene expression profiling of immunomagnetically separated cells directly from stabilized whole blood for multicenter clinical trials. Clin Transl Med. 2014;3:36.

32. Liberzon A. A description of the molecular signatures database (MSigDB) web site. Methods Mol Biol. 2014;1150:153-60.

33. Lipkin SM, Wang V, Jacoby R, Banerjee-Basu S, Baxevanis AD, Lynch HT, Elliott RM, Collins FS. MLH3: a DNA mismatch repair gene associated with mammalian microsatellite instability. Nat Genet. 2000;24(1):27-35.

34. Mackiewicz M, Shockley KR, Romer MA, Galante RJ, Zimmerman JE, Naidoo N, Baldwin DA, Jensen ST, Churchill GA, Pack Al. Macromolecule biosynthesis: a key function of sleep. Physiol Genomics. 2007;31(3):441-57.

35. Madabhushi R, Gao F, Pfenning AR, Pan L, Yamakawa S, Seo J, Rueda R, Phan TX, Yamakawa H, Pao PC, Stott RT, Gjoneska E, Nott A, Cho S, Kellis M, Tsai LH. Activity-induced DNA breaks govern the expression of neuronal early-response genes. Cell. 2015;161(7):1592-605.

36. Maret S, Dorsaz S, Gurcel L, Pradervand S, Petit B, Pfister C, Hagenbuchle O, O'Hara BF, Franken P, Tafti M. Homer1a is a core brain molecular correlate of sleep loss. Proc Natl Acad Sci U S A. 2007;104(50):20090-5.

37. McGinty DJ, Sterman MB. Sleep suppression after basal forebrain lesions in the cat. Science. 1968;160(3833):1253-5.

38. Moffitt JR, Bambah-Mukku D, Eichhorn SW, Vaughn E, Shekhar K, Perez JD, Rubinstein ND, Hao J, Regev A, Dulac C, Zhuang X. Molecular, spatial, and functional single-cell profiling of the hypothalamic preoptic region. Science. 2018;362(6416):eaau5324.

39. Mongrain V, Hernandez SA, Pradervand S, Dorsaz S, Curie T, Hagiwara G, Gip P, Heller HC, Franken P. Separating the contribution of glucocorticoids and wakefulness to the molecular and electrophysiological correlates of sleep homeostasis. Sleep. 2010;33(9):1147-57.

40. Morf J, Rey G, Schneider K, Stratmann M, Fujita J, Naef F, Schibler U. Coldinducible RNA-binding protein modulates circadian gene expression posttranscriptionally. Science. 2012;338(6105):379-83.

41. Naidoo N, Davis JG, Zhu J, Yabumoto M, Singletary K, Brown M, Galante R, Agarwal $B$, Baur JA. Aging and sleep deprivation induce the unfolded protein response in the pancreas: implications for metabolism. Aging Cell. 2014;13(1):131-41.

42. Nikonova EV, Gilliland JD, Tanis KQ, Podtelezhnikov AA, Rigby AM, Galante RJ, Finney EM, Stone DJ, Renger JJ, Pack Al, Winrow CJ. Transcriptional Profiling of Cholinergic Neurons From Basal Forebrain Identifies Changes in Expression of Genes Between Sleep and Wake. Sleep. 2017;40(6):zsx059.

43. Pinto RM, Dragileva E, Kirby A, Lloret A, Lopez E, Claire JS, Panigrahi GB, Hou C, Holloway K, Gillis T, Guide JR, Cohen PE, Li GM, Pearson CE, Daly MJ, Wheeler VC. Mismatch repair genes Mlh1 and Mlh3 modify CAG instability in Huntington's disease mice: genome-wide and candidate approaches. PLoS Genet. 2013;9(10):e1003930.

44. Ritchie ME, Phipson B, Wu D, Hu YF, Law CW, Shi W, Smyth GK. limma powers differential expression analyses for RNA-sequencing and microarray studies. Nucleic Acids Research. 2015;43(7):e47

45. Robinson MD, McCarthy DJ, Smyth GK. edgeR: a bioconductor package for differential expression analysis of digital gene expression data. Bioinformatics. 2010;26(1):139-40.

46. Saper CB, Chou TC, Scammell TE. The sleep switch: hypothalamic control of sleep and wakefulness. Trends Neurosci. 2001;24(12):726-31.

47. Schmittgen TD, Livak KJ. Analyzing real-time PCR data by the comparative C(T) method. Nat Protoc. 2008;3(6):1101-8.

48. Shen XH, Ellis RE, Sakaki K, Kaufman RJ. Genetic interactions due to constitutive and inducible gene regulation mediated by the unfolded protein response in C-elegans. PLoS Genet. 2005;1(3):355-68.
49. Sherin JE, Elmquist JK, Torrealba F, Saper CB. Innervation of histaminergic tuberomammillary neurons by GABAergic and galaninergic neurons in the ventrolateral preoptic nucleus of the rat. J Neurosci. 1998;18(12):4705-21.

50. Sherin JE, Shiromani PJ, McCarley RW, Saper CB. Activation of ventrolateral preoptic neurons during sleep. Science. 1996;271(5246):216-9.

51. Steininger TL, Gong H, McGinty D, Szymusiak R. Subregional organization of preoptic area/anterior hypothalamic projections to arousal-related monoaminergic cell groups. J Comp Neurol. 2001;429(4):638-53.

52. Suntsova N, Szymusiak R, Alam MN, Guzman-Marin R, McGinty D. Sleepwaking discharge patterns of median preoptic nucleus neurons in rats. $J$ Physiol. 2002;543(Pt 2):665-77.

53. Szymusiak R, Alam N, Steininger TL, McGinty D. Sleep-waking discharge patterns of ventrolateral preoptic/anterior hypothalamic neurons in rats. Brain Res. 1998;803(1-2):178-88.

54. Szymusiak R, Satinoff E. Ambient temperature-dependence of sleep disturbances produced by basal forebrain damage in rats. Brain Res Bull. 1984;12(3):295-305.

55. Taylor RC, Dillin A. XBP-1 is a cell-nonautonomous regulator of stress resistance and longevity. Cell. 2013:153(7):1435-47.

56. Thompson CL, Wisor JP, Lee CK, Pathak SD, Gerashchenko D, Smith KA, Fischer SR, Kuan CL, Sunkin SM, Ng LL, Lau C, Hawrylycz M, Jones AR, Kilduff TS, Lein ES. Molecular and anatomical signatures of sleep deprivation in the mouse brain. Front Neurosci. 2010;4:165.

57. Vecsey CG, Peixoto L, Choi JHK, Wimmer M, Jaganath D, Hernandez PJ, Blackwell J, Meda K, Park AJ, Hannenhalli S, Abel T. Genomic analysis of sleep deprivation reveals translational regulation in the hippocampus. Physiol Genomics. 2012;44(20):981-91.

58. von Ahlfen S, Missel A, Bendrat K, Schlumpberger M. Determinants of RNA quality from FFPE samples. PLoS One. 2007;2(12):e1261.

59. Wu D, Smyth GK. Camera: a competitive gene set test accounting for intergene correlation. Nucleic Acids Res. 2012;40(17):e133.

60. Xu Z, Cortes R, Villar M, Morino P, Castel MN, Hokfelt T. Evidence for upregulation of galanin synthesis in rat glial cells in vivo after colchicine treatment. Neurosci Lett. 1992;145(2):185-8.

61. Zada D, Bronshtein I, Lerer-Goldshtein T, Garini Y, Appelbaum L. Sleep increases chromosome dynamics to enable reduction of accumulating DNA damage in single neurons. Nat Commun. 2019;10(1):895.

62. Zhao S, Li Cl, Guo Y, Sheng Q, Shyr Y. RnaSeqSampleSize: real data based sample size estimation for RNA sequencing. BMC Bioinformatics. 2018;19(1): 191.

63. Zheng Q, Huang T, Zhang L, Zhou Y, Luo H, Xu H, Wang X. Dysregulation of ubiquitin-proteasome system in neurodegenerative diseases. Front Aging Neurosci. 2016:8:303

\section{Publisher's Note}

Springer Nature remains neutral with regard to jurisdictional claims in published maps and institutional affiliations.

Ready to submit your research? Choose BMC and benefit from

- fast, convenient online submission

- thorough peer review by experienced researchers in your field

- rapid publication on acceptance

- support for research data, including large and complex data types

- gold Open Access which fosters wider collaboration and increased citations

- maximum visibility for your research: over $100 \mathrm{M}$ website views per year

At BMC, research is always in progress.

Learn more biomedcentral.com/submissions 